\title{
Prognostic Factors and Association of Inflammatory Biomarkers with Severity and Mortality in COVID-19
}

\author{
Hamzullah Khan, Shahtaj Khan, Huma Riaz, Asad Rehman Khattak
}

ABSTRĀT'T:

Objectives: To determine prognostic values and association of inflammatory markers with severity and mortality in COVID19 in a hospital based study.

Study design and setting: This retrospective study was conducted from $1^{\text {st }}$ June 2020 to $30^{\text {th }}$ Sept 2020 in Department of Pathology, Qazi Hussain Ahmed Medical Complex, Nowshera and Medical Teaching Institute, Hayatabad Medical complex Peshawar.

Methodology: Out of 215, 71 cases were selected that had all relevant information's on chart available in Blood bank and department of Pathology.

Results: Out of 71 patients, 54 (76.1\%) were males and 17 (23.9\%) females. Thirty five (49.3\%) had age>55 years while $31(43.7 \%)$ were in age range 36-55 years. The frequency of the different blood groups were; 25 (35.2\%) B+ blood group followed by 19 (26.8\%) A+ and 14 (19.7\%) O+ blood group. The AUC for d-dimers was (0.725, 95\% CI 0.599-0.855) followed by CRP (0.565 95\%CI 0.422-0.7.8) and ferritin (0.519 95\%CI 0.36-0.679). The median values of d-dimers was significantly higher in the deceased as compared to the survivors ( $\mathrm{p}<0.05$ - Mann Whitney $\mathrm{U}$ test).The CRP and ferritin levels were not significantly different in study groups. There was a significant positive uphill correlation of the hospital stay with higher values of $d$-dimers $\left(\mathrm{p}=0.01, \mathrm{r}_{\mathrm{s}}=0.287\right)$.

Conclusion: D-dimer is a main prognostic factor that predicts mortality in COVID-19 followed by CRP and serum ferritin levels. Male gender and patient with age $>60$ are at risk of worst outcome under the impact of deranged values of inflammatory mediators. Hospital stay and blood group of patients have no relation with outcome.

Keywords: CRP, Mortality, COVID-19, Ferritin, D-dimers, Inflammatory markers.

How to cite this Article:

Khan H, Khan S, Riaz H, Khattak AR. Prognostic Factors and Association of Inflammatory Biomarkers with Severity and Mortality in COVID-19. J Bahria Uni Med Dental Coll. 2022; 12(1):25-30 DOI: https://doi.org/10.51985/JBUMDC054

This is an Open Access article distributed under the terms of the Creative Commons Attribution Non Commercial License (http:// creativecommons/org/licences/by-nc/4.0) which permits unrestricted non commercial use, distribution and reproduction in any medium, provided the original work is properly cited.

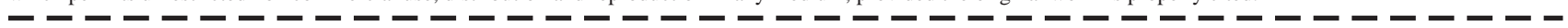

\section{INTRODUCTION:}

Covid-19 is a respiratory disease that was reported to the World Health Organization in Dec 2019 from the metropolitan city of Wuhan, China. The World Health Organization (WHO) declared global emergency due to rapid rise in cases of COVID-19 in China and nearby countries by the mid of

Hamzullah Khan,
Professor, Department of Hematology
I Eowshera Medical College, Nowshera/PGR HMC, Peshawar
I Email: hamzakmc@ gmail.com
Shahtaj Khan,
Professor, Department of Hematology
MTI Hayatabad Medical Complex, Peshawar
I Email: shahtajmasood@ gmail.com
Huma Riaz,
I Assistant Professor, Department of Hematology
MTI Hayatabad Medical Complex, Peshawar
I Email: humariaz82@yahoo.com
Asad Rehman Khattak
I Khdent, 4th years MBBS
I Received: 06-Sep-2021
I Accepted: 13-Dec-2021
February 2020. By the date $20^{\text {th }}$ Feb 2020 the number of laboratory confirmed cases reached to 60,000 in China with 1700 deaths. $^{1}$

Globally, Since the 31st December 2019 and till date $\left(22^{\text {nd }}\right.$ January2021), a total of 98,353,744 cases have been reported worldwide, in accordance with the definition of the Centre of Disease Control (CDC) of COVID-19 cases, with $2,106,818$ deaths. $^{2}$

In Pakistan, the literature so far covering the prevalence and incidence is deficient. By $22^{\text {nd }}$ Jan 2021, reported data from government sources declares 529,000 confirmed cases with 11204 deaths. Khyber Pukhtunkhwa has reported 64651 cases and 1823 deaths. $^{3}$

Increasing scientific research in field of COVID-19, the abnormalities in routine laboratory investigations, particularly the baseline investigations, has the potential to indicate the severity of the disease in a quick, easy, affordable, accessible and economical way. ${ }^{4}$ Consequently the inflammatory markers like serum ferritin, d-dimers and C-reactive proteins, prolactin and erythrocytes sedimentation rate have been reported to predict the disease severity and mortality in 
Prognostic Factors and Association of Inflammatory Biomarkers with Severity and Mortality in COVID-19

COVID-19. ${ }^{5}$ The number of patients are increasing in the second wave and critically ill patients needs ICU treatment that causing a major challenge. Therefore timely identification of severe disease is required with identification of values of prognostic markers to label patients with high risk for mortality.

Authors have reported from the city of Wuhan, China, the hyperferritnemia as predictor of mortality and severity of the disease. ${ }^{6}$ The literature supported the fact that on admission to isolation wards the average ferritin concentration in COVID-19 patients was >800 ìg/L, that was 5.3 times higher in patients with severe disease at time of presentation. Similarly in-hospital mortality was higher in patients with serum ferritin levels $>1400 \mathrm{ig} / \mathrm{L}$ as compared to the survivors with $<1400$ ig/L ferritin. ${ }^{7,8}$

A local study conducted in Peshawar, Pakistan concluded that more than $80 \%$ of the COVID-19 patients admitted in isolation and ICU had abnormally higher readings of serum ferritin. There was a statistically significant correlation of deranged ferritin levels with an increase in age of patient and with worst outcome in terms of death. Female gender more protective with higher chances of survival with deranged serum ferritin levels in all age groups compared to male gender. $^{9}$

Potentially the linkage of COVID-19 and venous thromboembolism is well described and reported by number of authors with significant alteration of the hematological parameters in corona infection. Some have described the positive association of mortality and morbidity with an increase in the level of d-dimers in COVID-19. ${ }^{10}$ A study reported the AUC for plasma CRP levels (0.896) on ROC curve which was significantly higher than age (0.833), ANC (0.820) and platelet count (0.677) in outcome prediction with cutoff values of 41.4 (Sensitivity $90 \%$, specificty $77 \%$ ). ${ }^{11}$

The rapid spread of the pandemic necessitates to prioritize patients in risk groups based on levels of these markers to detect the severity of the disease well in time to reduce mortality.

Facilities of costly cytokine analysis is not available everywhere in most of the healthcare facilities. Hence markers like CRP. D-dimers, ferritin are of great interst of the researcher for prognotic values to predict the severity of the pandemic.

Therefore present study was conducted to determine the prognostic values and association of inflammatory markers with severity and mortality in COVID-19 in a hospital based study

\section{METHODOLOGY:}

This retrospective study was conducted from $1^{\text {st }}$ June 2020 to $30^{\text {th }}$ Sept 2020 in Department of Pathology, Medical Teaching Institute (MTI) Qazi Hussain Ahmed Medical Complex, Nowshera and Postgraduate Medical Institute,
MTI, Hayatabad Medical complex Peshawar. Out of 215 files, 71 COVID-19 patients had complete information and they were referred to pathology with the request of transfusion of packed cells, blood product, FFP or COVID convalescent plasma therapy' were further studied.

Patients hospitalized with COVID-19 in ICU-COVID-19, HMC were included. Patients where all inflammatory mediators were advised like d-dimers, ferritin, and CRP were advised by the treating consultant were included only, irrespective of age and gender. Similarly those patients were further observed for disease outcome in terms of satisfactory discharge or case fatality due to COVID-19 to be included.

COVID-19 patients where deficient inflammatory mediator readings were available on chart of patients, or patients expired before to be fully investigated were excluded. Similarly all patients with any type of symptoms came to emergency or outdoor patients department or even confirmed patients of COVID-19 in isolation department where readings of the inflammatory mediators were not advised were also excluded. The Serum Ferritin, d-dimer levels was measured by electro-chemiluminescence immunoassay using Roche Cobas E411 Chemistry Analyzer, using commercial kits of Roche diagnostics as per the instructions of the manufacturer.

This study was conducted in line with the research regulations, followed the sound medical practice, redetected human rights and also within the principles of declaration of Helsinki of World Medical Association.

Ethical endorsement was obtained from the institutional ethical review board of Post-Graduate Medical Institute, Hayatabad Medical Complex under notification No (316/HEC/B \& PSC/2020 Dated $15^{\text {th }}$ May 2020) and notification No (346/HEC/B \& PSC/2020 Dated $10^{\text {th }}$ Dec 2020).

For COVID confirmation, PCR results of the naso-pharyngeal swab duly reported in Public health research laboratory of the Hospital and also of the Khyber medical University (a designated Lab for RT-PCR of 2019nCoV by the Government of Khyber Pukhtunkhwa) were considered only.

Data was entered in SPSS, version 25 and descriptive and correlation statistics were applied. Numerical variables like age of patients and serum ferritin, d-dimer, CRP, age and hospital stay were presented with Mean and SD in case of normal distribution and with median and range where the data was not normally distributed.

Normality of data was assessed using Shapiro-wilk Test.

Independent t-test/Mann Whitney $U$ test were used to determine the difference of the numerical variables (serum ferritin, d-dimer, CRP, age and hospital stay) in groups (discharged satisfactory vs expired). Chi-square test was used to show an association of blood group age and gender with disease outcome in COVID-19 patients.

Receiver operating characteristics (ROC) curve was used 
to determine the relationship of clinical sensitivity and specificity of different inflammatory mediators to predict the worst outcome in COVID-19. Correlation tests using Spearman ranked correlation was used to determine the correlation of quantitative variables (serum ferritin d-dimer, CRP, age and hospital stay).

\section{RESULTS:}

Out of 71 patients who were selected for plasma therapy, $54(76.1 \%)$ were males and $17(23.9 \%)$ were females. Thirty five $(49.3 \%)$ had age $>55$ years while $31(43.7 \%)$ were in age range $36-55$ years and $5(7 \%)$ had age less than 35 years. Majority 25 (35.2\%) had B+ blood group followed by 19 (26.8\%) A+, $14(19.7 \%)$ O+ blood groups. Sixty one $(85.91 \%$ had Rh+ while $10(14.08 \%)$ had Rh- blood. Out of total, $25(35 \%)$ expired during hospitalization.

The data distribution was assessed with Shapirowilk test and we found that distribution of age in both genders was normally distributed ( $\mathrm{p}$-value>0.05) while the distribution of all the three inflammatory markers was in a skewed pattern ( $p$-value<0.05).

While assessing the association of different demographic and hematological factors with outcome, we observed that there was no significant association of gender (p-0.993), age (0.892) and blood group (0.855).

The difference in Mean/median values of different inflammatory markers and hospital stay with disease outcome, it was observed that the median value of d-dimers was significantly higher in the deceased as compared to the survivors ( $p<0.05$ - Mann Whitney $U$ test). No significant difference in median value of ferritin was observed between survivors versus non-survivors (Mann Whitney U test, $\mathrm{p}>0.05)$. The Mean with SD of CRP and hospital stay was not significantly different in groups (survivors vs expired) with (independent t test, $\mathrm{p}=0.566, \mathrm{p}=0.423$ respectively). (Table 1)

The cut off values of inflammatory mediators for predicting mortality is shown in Table 2. The table further shows the AUC for d-dimers was $(0.725,95 \%$ CI 0.599-0.855) followed by CRP (0.565 95\%CI 0.422-0.7.8) and ferritin (0.519 95\%CI 0.36-0.679).

Correlation matrix showing the correlation coefficient for different quantitative variables in table 6. There was a positive uphill significant correlation of hospital stay with higher values of $d$-dimers $\left(p=0.01, r_{s}=0.287\right)$. Unlikely $d-$ dimer by assessing the correlation of the hospital stay with CRP and ferritin was not statistically significant. (Table 3)

The ROC curve showing the area under curve for different inflammatory markers in figure 1.

\section{DISCUSSION:}

Male predominance $(76.1 \%)$ was found in this study. Likewise $35(49.3 \%)$ of the patients presented with age $>55$ years and $25(35.2 \%)$ had B+ blood group followed as major

Table 1. Difference in inflammatory markers in COVID-19 in study groups

\begin{tabular}{|l|c|c|c|c|}
\hline Hematological Markers & $\begin{array}{c}\text { Improved or (survivors) } \\
(\mathbf{N = 4 2})\end{array}$ & $\begin{array}{c}\text { Died or (non-survivors) } \\
(\mathbf{N = 2 5})\end{array}$ & $\mathbf{p}$-value & Test of significance \\
\hline $\begin{array}{l}\text { C-Reactive Protien (CRP) } \\
\text { Mean } \pm \text { SD }\end{array}$ & $15.18 \pm 12.10$ & $16.86 \pm 10$ & 0.566 & Independent T Test \\
\hline $\begin{array}{l}\text { Serum Ferritin } \\
\text { Median }\end{array}$ & 1518.4 & 1363.12 & $\mathrm{p}>0.05$ & Mann Whitney U Test \\
\hline $\begin{array}{l}\text { d-dimers } \\
\text { Median }\end{array}$ & 28.89 & 135.4 & $\mathrm{p}<0.05$ & Mann Whitney U Test \\
\hline $\begin{array}{l}\text { Hospital Stay } \\
\text { Mean } \pm \text { SD }\end{array}$ & $9.6 \pm 8$ & $7.92 \pm 8$ & 0.423 & Independent T Test \\
\hline
\end{tabular}

Table 2. ROC statistics for predicting mortality in COVID-19 patients

\begin{tabular}{|c|c|c|c|c|c|c|c|c|}
\hline & & & & & \multicolumn{2}{c|}{$\begin{array}{c}\text { Asymptotic 95\% } \\
\text { Confidence Interval }\end{array}$} & \\
\hline Test Result Variable(s) & $\begin{array}{c}\text { Cutoff } \\
\text { values }\end{array}$ & $\begin{array}{c}\text { Sensitivity } \\
(\%)\end{array}$ & $\begin{array}{c}\text { Specificity } \\
(\%)\end{array}$ & $\begin{array}{c}\text { Area } \\
(\text { AUC })\end{array}$ & Std. Error & $\begin{array}{c}\text { Lower } \\
\text { Bound }\end{array}$ & $\begin{array}{c}\text { Upper } \\
\text { Bound }\end{array}$ & Sig. (p-value) \\
\hline D_Dimer & 2.25 & 82 & 80 & 0.725 & 0.064 & 0.599 & 0.85 & 0.002 \\
\hline CRP & 8.7 & 72 & 57 & 0.565 & 0.073 & 0.422 & 0.708 & 0.378 \\
\hline Ferritin & 1103 & 62 & 60 & 0.519 & 0.081 & 0.36 & 0.679 & 0.795 \\
\hline $\begin{array}{l}\text { The test result variable(s): D_Dimer, CRP, Ferritin has at least one tie between the positive actual state group and the } \\
\text { negative actual state group. Statistics may be biased. }\end{array}$ \\
a. Under the nonparametric assumption \\
b. Null hypothesis: true area = 0.5 \\
\hline
\end{tabular}


Prognostic Factors and Association of Inflammatory Biomarkers with Severity and Mortality in COVID-19

Table 3. Correlation matrix showing the the Spearman's correlation coefficient (rs) for Hospital stay with different inflammatory markers

\begin{tabular}{|l|c|l|l|c|}
\hline \multicolumn{2}{|c|}{} & CRP & Ferritin & D_Dimer \\
\hline \multirow{2}{*}{ Ferritin } & $\mathbf{r}_{\mathbf{s}}$ & 0.196 & & \\
\cline { 2 - 5 } & $\mathbf{p}$ & 0.111 & & \\
\hline \multirow{2}{*}{ D_Dimers } & $\mathbf{r}_{\mathbf{s}}$ & 0.103 & 0.189 & \\
\cline { 2 - 5 } & $\mathbf{p}$ & 0.405 & 0.123 & \\
\hline $\begin{array}{l}\text { Hospital stay } \\
\text { (days) }\end{array}$ & $\mathbf{r}_{\mathbf{s}}$ & 0.037 & 0.044 & $.287^{*}$ \\
\cline { 2 - 5 } & $\mathbf{p}$ & 0.764 & 0.719 & 0.017 \\
\hline Number of patients & 67 & 68 & 68 \\
\hline
\end{tabular}

Figure 1. ROC Curve for inflammatory markers
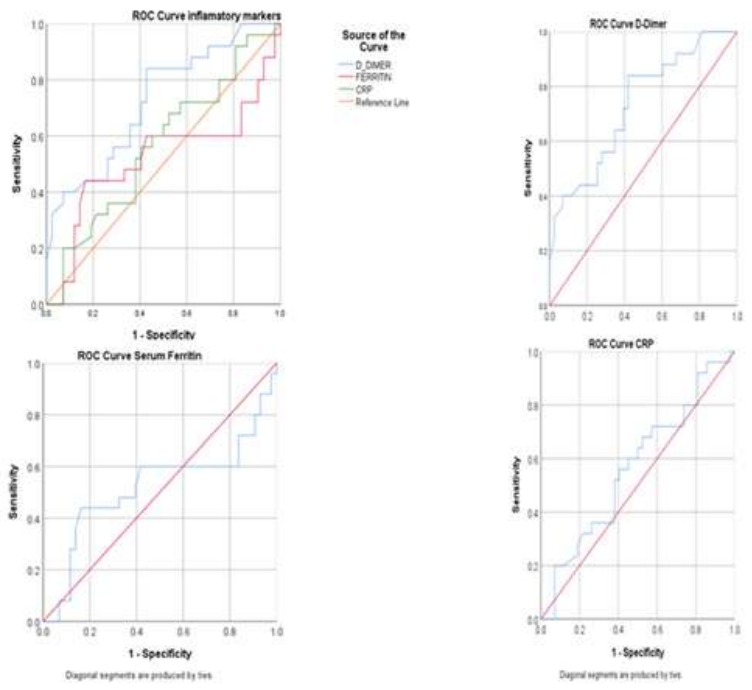

blood groups. The target population was bit biased as included patients who were selected for plasma therapy and by default in initial phases only serious patients were selected for plasma therapy and was thought to be one of the most safe therapy for the serious patients. Total $n=25$ cases out of 71 of the target population whose files were present in the department reported expired during hospitalization. This mortality reflects the higher rate and as mainly of those patients had serious disease. Reportedly 215 cases received transfusion of blood products for different complication and needs. It was observed that the AUC for d-dimers was (0.725) followed by CRP $(0.565)$ and ferritin (0.519) which shows the higher clinical sensitivity of d-dimer on ROC curve to predict mortality in COVID-19. Similarly the median value of d-dimers was significantly higher in the deceased as compared to the survivors (28 vs 135 ) with pvalue $<0.05$. The surviving ability drops to less than $20 \%$ in age $>70$ with female gender confer more immunity/chances of survival as compared to male gender.

The measurements of inflammatory markers assist the physicians to predict the severity of the disease. ${ }^{12}$ Accumulative assessment have concluded that inflammatory response play a critical role in the COVID-19 progression. ${ }^{12,13}$
Several inflammatory markers such as Serum ferritin, ddimers, c-reactive proteins, Interleukin- 6 have been reported with high risk of the severe disease. ${ }^{13}$

Gao $\mathrm{Y}$ et $\mathrm{al}^{14}$ have reported that the area under curved for deranged d-dimers as 0.840, which is higher than the findings and further confers the higher clinical impact of deranged d-dimers on the worst outcome in COVID-19. Similarly a meta-analysis reported d-dimer 3.9 times higher in the exposed groups who meet worst outcome in term of death. ${ }^{15}$ The abnormally deranged serum ferritin levels may be crucial for in COVID-19 patients. A study from Wuhan reported patients who had d-dimer $>2 \mu \mathrm{g} / \mathrm{mL}$ had higher fatality than patient who presented with d-dimer $>2 \mu \mathrm{g} / \mathrm{mL}$ $(\mathrm{p}<0.05) .16$

A study appeared in JAMA recommended therefore that it would be more beneficial to use the cut off values of the ddimers for specific population and then for age and gender in the scenario of the COVID-19. That must be added then to the national surveillance system to be used for population studies to determine the high risk group more vulnerable to meet worst outcome in COVID-19. ${ }^{17}$

A local study reported the probability of worse outcome in term of death was 2.06 times more in patients with dimers $>0.5 \mu \mathrm{g} / \mathrm{ml}(\mathrm{OR}=2.06)$ with a relative risk of $1.1 .^{18}$

The serum ferritin levels are reported high and non stoppable in hospitalized patient with COVID-19. A study reported an increase in the ferritin levels exceeding the upper limit of detection, with increase in hospital stay. Similarly ferritin levels were significantly higher $(\mathrm{p}<0.05)$ in critically ill patients (ICU patients) as compared to severe diseased group (Non-ICU). ${ }^{19}$ However in present study it was observed a higher ferritin in both groups survivors vs. deceased and was not statistically different in both the groups. The reason may be that hospital is the end stage referral hospitals for COVID-19 care and usually received referral at very end stage with severe disease. A study from China reported higher levels of the serum ferritin in patients died due to COVID-19 infection as compared to the patients discharged. But a trend of high ferritin was noted in COVID-19 patients throughout the clinical course as observed in both the groups (Discharges vs Deceased). ${ }^{20}$ Another local study that was reported from the Peshawar Pakistan showed that $80 \%$ of the COVID-19 patients had deranged levels of serum ferritin at presentation to hospital. These abnormally higher ferritin levels had a statistically significant relationship with an increase in age of patients and outcome in terms of death. ${ }^{9}$

Furthermore it was the only hospital that was allowed and authorized for plasma exchange and included patients from ICU. Therefore at entry to the ICU, majority of the patients were in cytokine storm with high inflammatory markers. Hence, ferritin values was higher in both groups irrespective of outcome and the impact on outcome was less specific than d-dimer. High d-dimer values can be observed in severe 
disease after long time while the values of the ferritin and d-dimer decreased with an increase in the disease duration. Likewise a higher in-hospital mortality has been reported in patient with high levels of CRP $(>101 \mathrm{mg} / \mathrm{dl}) .{ }^{20}$ Their findings were higher than the results of this study where the mean $+\mathrm{SD}$ of CRP was $16.86+10 \mathrm{mg} / \mathrm{dl}$, but the difference was not statistically significant.

A retrospective study from Karachi reported C-reactive protein (CRP) levels predicting ICU admission with area under the curve (AUC): 0.806, positive predictive value (PPV): $85.1 \%{ }^{21}$

Thus findings of this study support the literature reported from the metropolitan cities affected by COVID-19. The initial readings of the inflammatory markers are predictive of severity and mortality for this pandemic, however the preference in values of d-dimer with ferritin and CRP is different in different studies reported from different quarter/regions as evident from literature/research studies. ${ }^{16,}$ 19, 21,22 These markers values need to be specified for different age and gender groups of the respective populations in order to know the severity of the disease. There were some limitations of the study. Smaller sample size restricts us to predict the application of this study on larger population, including more inflammatory markers like LDH etc. Studies executed with larger sample size can estimate the true population impact. Therefore it is suggested that future studies should cover large population with representation to predict/suggest the findings for decision makers.

\section{CONCLUSION:}

D-dimer values can be relied as best prognostic indicator at all stages of the disease. D-dimer is a main prognostic factor that predicts mortality in COVID-19 followed by CRP and serum ferritin levels. Male gender and patient with age $>60$ are at risk of worst outcome under the impact of deranged values of inflammatory mediators. Hospital stay itself has no significant correlation with outcome. Blood group itself does not predict about the severity or fatality of the disease.

Hamzullah Khan: Conception, design, analysis and/or I interpretation of data writing the manuscript

I Shahtaj Khan: Design, Data analysis and critical review

Huma Riaz: Data Collection analysis and/or interpretation of I data

I Asad Rehman Khattak: Data Collection

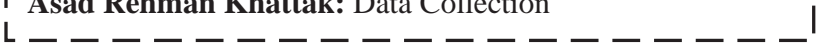

\section{REFERENCES:}

1. World Health Organization. 2019-nCoV outbreak is an emergency of international concern. 2020. http://www.euro. who.int/en/health- topics/emergencies/pages/news /news/2020/01/2019-ncov-outbreak-is-an-emergency-ofinternational-concern (access Feb 16, 2020).

2. Centre of Disease Control-Confirmed cases. https://www .ecdc.europa.eu/en/geographical-distribution-2019-ncov-cases. Retrieved 28th Nov 2020.
3. "Coronavirus in Pakistan - Confirmed Cases". www.covid .gov.pk/. Retrieved 22nd Jan 2021.

4. Usul E, Pan Ý, Bekgöz B, Pahin A. Role of hematological parameters in COVID-19 patients in the emergency room. Biomark Med. 2020;14(13):1207-1215. doi:10.2217/bmm2020-0317

5. Gómez-Pastora J, Weigand M, Kim J, et al. Hyperferritinemia in critically ill COVID-19 patients - Is ferritin the product of inflammation or a pathogenic mediator? Clin Chim Acta. 2020;509:249-51. https://doi:10.1016/j.cca.2020.06.033

6. Chen G, Wu D, Guo W, Cao Y, Huang D, Wang $\mathrm{H}$ et al. Clinical and immunological features of severe and moderate coronavirus disease 2019. J Clin Invest. 2020;130(5):262029. https://doi.org/ 10.1172/J CI137244

7. Bai T, Tu S, Wei Y, Xiao L, Jin Y, Zhang L, et al Clinical and Laboratory Factors Predicting the Prognosis of Patients with COVID-19: An Analysis of 127 Patients in Wuhan, China (2/26/2020). Available at SSRN: https://ssrn. com/ abstract= 3546118 or http://dx.doi .org/10.2139/ ssrn. 3546118

8. Zhou F, Yu T, Du R, Fan G, Liu Y, Liu Z, Xiang J et al. Clinical course and risk factors for mortality of adult inpatients with COVID-19 in Wuhan, China: a retrospective cohort study. Lancet. 2020; 395:1054-62.

9. Khan S, Zahoor S, Khan H, Khan K, Farooq M. Hyperferritinemia as independent risk factor and biomarker in COVID-19 to predict severity and mortality. Professional Med J 2021; 28(8):1183-1189. https://doi.org/ 10.29309/ TPMJ/2021.28.08.6178

10. Zhou F, Yu T, Du R. Clinical course and risk factors for mortality of adult in patients with COVID-19 in Wuhan, China: a retrospective cohort study. Lancet. 2020, 28;395(10229):1054-1062. https://doi: 10.1016/S01406736(20)30566-3.

11. Luo X, Zhou W, Yan X, Guo T, Wang B, Xia H, et al. Prognostic Value of C-Reactive Protein in Patients With Coronavirus 2019. Clin Infect Dis. 2020 Nov 19;71(16):217479.

12. Zeng F, Huang Y, Guo Y, Yin M, Chen X, Xiao L, Deng G. Association of inflammatory markers with the severity of COVID-19: A meta-analysis. Int J Infect Dis. 2020;96:467474.

13. Stebbing J, Phelan A, Griffin I., Tucker C, Oechsle O, Smith D. COVID-19: combining antiviral and anti-inflammatory treatments. Lancet Infect Dis. 2020;20(4):400-402.

14. Gao Y, Li T, Han M, Li X, Wu D, Xu Y, et al. Diagnostic utility of clinical laboratory data determinations for patients with the severe COVID-19. J Med Virol 2020;92(7):791-6.

15. Ishazli RM, Toraih EA, Elgaml A, El-Mowafy M, El-Mesery M, Amin MN, et al. Diagnostic and prognostic value of hematological and immunological markers in COVID-19 infection: A meta-analysis of 6320 patients. PLoS One. 2020;15(8):e0238160.

16. Zhang L, Yan X, Fan Q, Liu H, Liu X, Liu Z, Zhang Z. Ddimer levels on admission to predict in-hospital mortality in patients with Covid-19. J Thromb Haemost. 2020 Jun;18 (6): 1324-1329. https://doi: 10.1111/jth.14859.

17. Onder G, Rezza G, Brusaferro S. Case-Fatality rate and characteristics of patients dying in relation to COVID-19 in Italy. JAMA. 2020;323(18):1775-1776. https://doi: 10. 1001/jama.2020.4683 
Prognostic Factors and Association of Inflammatory Biomarkers with Severity and Mortality in COVID-19

18. Khan S, Khan H, Khan S, Akhtar M. Evaluation of variation of D-dimer levels in COVID-19 patients to predict the disease outcome in a hospital based study. Anaesth. pain intensive care 2020;24(5):490-496

19. Zhonghua Liu Xing Bing Xue ZaZhi. Novel coronavirus pneumonia emergency response epidemiology team. Prime PubMed, 2020; 41(2):145-51. https://doi: 10.3760/cma.j.issn. 0254-6450.2020.02.003.

20. Ullah W, Thalambedu N, Haq S, Saeed R, Khanal S, Tariq S, et al. Predictability of CRP and D-Dimer levels for in-hospital outcomes and mortality of COVID-19. J Community Hosp Intern Med Perspect. 2020;10(5):402-8.
21. Asghar MS, Haider Kazmi SJ, Ahmed Khan N, Akram M, Ahmed Khan S, Rasheed U, Hassan M, Memon GM. Clinical Profiles, Characteristics, and Outcomes of the First 100 Admitted COVID-19 Patients in Pakistan: A Single-Center Retrospective Study in a Tertiary Care Hospital of Karachi. Cureus. 2020 Jun 20;12(6):e8712. https://doi: 10.7759/ cureus. 8712.

22. Lin Z, Long F, Yang Y, Chen X, Xu L, Yang M. Serum ferritin as an independent risk factor for severity in COVID-19 patients. J Infect. 2020 Oct;81(4):647-679. https://doi doi: 10.1016/j.jinf.2020.06.053.

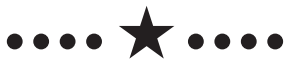

